\title{
A DETERMINANT OF THE CHUDNOVSKYS GENERALIZING THE ELLIPTIC FROBENIUS-STICKELBERGER-CAUCHY DETERMINANTAL IDENTITY
}

\author{
Tewodros Amdeberhan \\ Mathematics, DeVry Institute of Technology, North Brunswick, NJ 08902, USA \\ amdberha@nj.devry.edu, tewodros@math.temple.edu
}

Submitted: October 16, 2000. Accepted: October 23, 2000.

\begin{abstract}
D.V. Chudnovsky and G.V. Chudnovsky [CH] introduced a generalization of the FrobeniusStickelberger determinantal identity involving elliptic functions that generalize the Cauchy determinant. The purpose of this note is to provide a simple essentially non-analytic proof of this evaluation. This method of proof is inspired by D. Zeilberger's creative application in [Z1].
\end{abstract}

AMS Subject Classification: Primary 05A, 11A, 15A

One of the most famous alternants is the Cauchy determinant which is only a special case of a determinant with symbolic entries:

$$
\operatorname{det}\left[\frac{1}{x_{i}-y_{j}}\right]_{1 \leq i, j \leq n}=(-1)^{n(n-1) / 2} \frac{\prod_{i<j}\left(x_{i}-x_{j}\right)\left(y_{i}-y_{j}\right)}{\prod_{i=1}^{n} \prod_{j=1}^{n}\left(x_{i}-y_{j}\right)} .
$$

This expression lends itself to explicit formulas in Padé approximation theory and further applications in transcendental theory. On the other hand, the Cauchy determinant cannot be readily generalized to trigonometric or elliptic functions. However, its associate can.

A natural elliptic generalization of the $1 / x$ Cauchy kernel to the corresponding Riemann surface would be the Weierstraß $\zeta$-function. Such a generalization was supplied by Frobenius and Stickelberger [FS], with references given to Euler and Jacobi.

D.V. Chudnovsky and G.V. Chudnovsky $[\mathrm{CH}]$ introduced a generalization of the Frobenius Stickelberger determinantal identity involving elliptic functions that generalizes the Cauchy determinant.

The purpose of this note is to provide a simple essentially non-analytic proof of this evaluation. This method of proof is inspired by D. Zeilberger's creative application in [Z1].

We begin by recalling some notations. Given the Weierstraß elliptic function, $\wp(z)$, then the Weierstraß $\zeta$-function and $\sigma$-function are defined respectively by

$$
\wp(z)=-\frac{d}{d z} \zeta(z), \quad \text { and } \quad \zeta(z)=\frac{d}{d z} \log \sigma(z)
$$


Theorem $[\mathbf{C H}]$ : For arbitrary $n \geq 1$ we have

$$
\begin{gathered}
\operatorname{det}\left[\frac{\sigma\left(u_{i}+v_{j}+e\right)}{\sigma\left(u_{i}+v_{j}\right) \sigma(e)} e^{\gamma_{1} u_{i}+\gamma_{2} v_{j}}\right]_{1 \leq i, j \leq n} \\
=\frac{\sigma\left(\sum u_{i}+\sum v_{j}+e\right) \prod_{i>j} \sigma\left(u_{i}-u_{j}\right) \sigma\left(v_{i}-v_{j}\right)}{\sigma(e) \prod_{i, j=1}^{n} \sigma\left(u_{i}+v_{j}\right)} e^{\gamma_{1} \sum u_{i}+\gamma_{2} \sum v_{j}},
\end{gathered}
$$

where $u_{i}, v_{j}$ and $e$ are arbitrary parameters on the elliptic curve.

First, we prove a lemma (set $a=b=0$ to get the result of the theorem).

Lemma: With the additional parameters $a$ and $b$, we have

$$
\begin{gathered}
\operatorname{det}\left[\frac{\sigma\left(u_{i+a}+v_{j+b}+e\right)}{\sigma\left(u_{i+a}+v_{j+b}\right) \sigma(e)} e^{\gamma_{1} u_{i+a}+\gamma_{2} v_{j+b}}\right]_{1 \leq i, j \leq n} \\
=\frac{\sigma\left(\sum u_{i+a}+\sum v_{j+b}+e\right) \prod_{i>j} \sigma\left(u_{i+a}-u_{j+b}\right) \sigma\left(v_{i+a}-v_{j+b}\right)}{\sigma(e) \prod_{i, j=1}^{n} \sigma\left(u_{i+a}+v_{j+b}\right)} e^{\gamma_{1} \sum u_{i+a}+\gamma_{2} \sum v_{j+b}} .
\end{gathered}
$$

Proof: Let the left and right sides of equation (4) be $L_{n}(a, b)$ and $R_{n}(a, b)$, respectively. Dodgson's rule [D] (see [Z2] for a bijective proof) for evaluating determinants immediately implies [Z1] the recurrence Lewis:

$$
X_{n}(a, b)=\frac{X_{n-1}(a, b) X_{n-1}(a+1, b+1)-X_{n-1}(a+1, b) X_{n-1}(a, b+1)}{X_{n-2}(a+1, b+1)}
$$

holds with $X=L$. Moreover, the same is true if $X=R$. Indeed the latter takes the form of a "three-term recurrence"

$$
\begin{aligned}
\sigma\left(A_{1}+A_{2}\right) \sigma\left(A_{1}-A_{2}\right) \sigma\left(A_{4}+A_{3}\right) \sigma\left(A_{4}-A_{3}\right) & =\sigma\left(A_{4}+A_{1}\right) \sigma\left(A_{4}-A_{1}\right) \sigma\left(A_{3}+A_{2}\right) \sigma\left(A_{3}-A_{2}\right) \\
& -\sigma\left(A_{3}+A_{1}\right) \sigma\left(A_{3}-A_{1}\right) \sigma\left(A_{4}+A_{2}\right) \sigma\left(A_{4}-A_{2}\right),
\end{aligned}
$$

where

$$
\begin{gathered}
y:=\sum_{i=2}^{n-1}\left(u_{a+i}+v_{b+i}\right), \quad w:=\left(y+u_{a+1}+u_{b+n}\right) / 2, \quad A_{1}:=w-u_{a+1}, \\
A_{2}:=w-u_{a+n}, \quad A_{3}:=w+v_{b+1} \quad \text { and } \quad A_{4}:=w+v_{b+n} .
\end{gathered}
$$

Equation (5) is similar to the well-known Jacobi identity on $\sigma$-functions (this is due to Weierstraß, in lectures by Schwarz [S] p. 47):

$$
\begin{aligned}
\sigma(z+a) \sigma(z-a) \sigma(b+c) \sigma(b-c) & +\sigma(z+b) \sigma(z-b) \sigma(c+a) \sigma(c-a) \\
& +\sigma(z+c) \sigma(z-c) \sigma(a+b) \sigma(a-b)=0
\end{aligned}
$$

and both equations follow from $\theta$-functions identities or the "parallelogram" identity

$$
\wp(z)-\wp(y)=-\frac{\sigma(z+y) \sigma(z-y)}{\sigma(z)^{2} \sigma(y)^{2}} .
$$


In fact, a repeated application of (6) in the former equation leads to a trivial algebraic equation in cyclic notations

$$
\begin{aligned}
\left(\wp\left(A_{1}\right)-\wp\left(A_{2}\right)\right)\left(\wp\left(A_{4}\right)-\wp\left(A_{3}\right)\right) & -\left(\wp\left(A_{4}\right)-\wp\left(A_{1}\right)\right)\left(\wp\left(A_{3}\right)-\wp\left(A_{2}\right)\right) \\
& +\left(\wp\left(A_{3}\right)-\wp\left(A_{1}\right)\right)\left(\wp\left(A_{4}\right)-\wp\left(A_{2}\right)\right)=0 .
\end{aligned}
$$

Since $L_{n}(a, b)=R_{n}(a, b)$ for $n=1$ (trivial!), and $n=2$ (check!), it follows by induction that

$$
L_{n}(a, b)=R_{n}(a, b) \quad \text { for all } n
$$

\section{REFERENCES}

[CH] D.V. Chudnovsky, G.V. Chudnovsky, Hypergeometric and modular function identities, and new rational approximations and continued fraction expansions of classical constants and functions, Contemporary Math. 143 (1993), 117-162.

[D] C.L. Dodgson, Condensation of Determinants, Proc. Royal Soc. of London 15 (1866), 150-155.

[FS] F. Frobenius, L. Stickelberger, Uber die Addition und Multiplication der elliptischen Functionen, F. Frobenius, Gesammelte Abhandlungen, B. I (1968), Springer, New York, 612-650.

[S] H. A. Schwarz, Formeln und Lehrsätze zum Gebrauche der elliptichen Funktionen, Vorlesungen und Aufzeichnungen des Herrn Prof. K. Weierstrass, Berlin, 1893.

[Z1] D. Zeilberger,, Reverend Charles to the aid of Major Percy and Fields Medalist Enrico, Amer. Math. Monthly 103 (1996), 501-502.

[Z2] D. Zeilberger,, Dodgson's Determinant-Evaluation Rule Proved by TWO-TIMING MEN and WOMEN, Elec. J. Comb. [Wilf Festchrifft] 4 (2) \#R22 (1997). 